\title{
Psicooncología
}

ISSN: $1696-7240$

http://dx.doi.org/10.5209/PSIC.61453

\section{Estudio sobre el duelo en psicooncología pediátrica}

Autor: Valeria Moriconi

Tipo de trabajo: Tesis doctoral

Director: Dr. Luis Madero López

Centro de Investigación: Hospital Universitario Niño Jesús. Universidad Autónoma de Madrid.

Fecha de Lectura: 22 de junio de 2018.

E-mail: valemoric@hotmail.com

\section{Resumen}

Introducción: la temática del duelo de los padres ha recibido la atención de los investigadores solo en los últimos años, cuando se hizo evidente que esta realidad difiere sustancialmente del proceso de duelo general, analizado por la literatura psicológica moderna.

Este trabajo tiene como fin evidenciar las necesidades de las personas en duelo; aumentar la especificidad y la eficacia del abordaje terapéutico para prevenir el riesgo de que el proceso se complique; y analizar la evolución de las personas afligidas en el tiempo y su adecuación a la pérdida.

Método: en este estudio hemos creído oportuno utilizar un enfoque cualitativo ya que buscamos comprender la perspectiva de los participantes acerca del fenómeno del duelo.

Se han analizado las sesiones de 5 grupos terapéuticos de elaboración del duelo por un total de 111 sesiones. Los 111 files de las transcripciones han sido elaborados con el software SAS Text Mining para crear las tablas de datos y poder hacer el análisis, seguidamente se ha utilizado el nudo Análisis de Topic para delinear núcleos temáticos claros y válidos.

En una segunda fase, para garantizar una mayor fiabilidad y validez del estudio, hemos realizado varios Focus Group. De los 50 participantes a los grupos de duelo 32 personas se demostraron disponibles a acudir a los Focus Group. Se organizaron seis grupos el número de participantes de cada uno de los grupos fueron entre cuatro y ocho por grupo.

Los padres que participaron lo hicieron desde la doble motivación de ayudar a ampliar el conocimiento científico acerca de las dinámicas del duelo y de ayudar a otras familias en situaciones semejantes de duelo. 
Resultados: analizando los datos relativos a las sesiones terapéuticas de los grupos de duelo hemos podido evidenciar cinco núcleos temáticos bien definidos y no confluyentes entre si. Hemos denominado los topic Desahogo Emocional, Herramientas, Asuntos Pendientes, Legado y Sentido.

En los Focus Group los padres reconocen en los núcleos temáticos el trabajo realizado. Destacan como ejes más eficaces al fin de la elaboración del duelo el Desahogo Emocional, el Legado y las Herramientas, el Sentido y en mínima parte los Asuntos Pendientes. Los padres afirman con seguridad que la experiencia terapéutica grupal ha sido de ayuda en la elaboración del duelo y en la reconstrucción de la identidad a la que se han enfrentado tras la muerte de sus hijos. En el tiempo los cambios logrados en la terapia han persistido e incluso evolucionado hacia un vivir más conforme a los nuevos valores e ideales.

Conclusiones: este estudio quiere servir de ayuda para comprender el difícil mecanismo que se pone en marcha en los padres cuando muere un hijo, qué es lo que necesitan y cómo se les puede proporcionar con intervenciones efectivas y oportunas.

Las implicaciones que este estudio tiene en la practica clínica son útiles no solo en el ámbito del duelo inherente a la muerte si no también en el trabajo del duelo entendido como perdida. Por lo tanto conocer los núcleos temáticos sobre los que se mueve la elaboración psíquica del trauma es relevante en todas las fases de la enfermedad y para todos los profesionales implicados en el tratamiento de la misma.

Conocer los mecanismos subyacentes a la elaboración de la perdida no solo previene sufrimiento psicológico también incrementa la calidad humana del cuidado hacia los pacientes. 